\title{
Evaluación de Protocolos de Señalización en Multiprotocolo Generalizado de Conmutación de Etiquetas (GMPLS) Capaces de Conmutar Paquetes y Longitudes de Onda
}

\author{
Elkin M. Piedrahita, Cesar A. Hernández*, Luis F. Pedraza, Octavio J. Salcedo \\ Universidad Distrital "Francisco José de Caldas", Carrera 7 No. 40 - 53 Bogotá-Colombia, \\ (e-mail: empiedrahitav@correo.udistrital.edu.co, cahernandezs@udistrital.edu.co, \\ Ifpedrazam@udistrital.edu.co, ojsalcedop@udistrital.edu.co) \\ *Autor a quien debe ser dirigida la correspondencia.
}

Recibido May. 23, 2013; Aceptado Jul. 29, 2013; Versión final recibida Sep. 24, 2013

\section{Resumen}

En este artículo, se evalúan los protocolos de señalización RSVP-TE y CR-LDP en el Multiprotocolo Generalizado de Conmutación de Etiquetas (GMPLS), con base en los parámetros de diseño de la capa física, y las conmutaciones de paquetes y longitudes de onda. En las simulaciones de los protocolos de señalización los resultados no presentan mayor discrepancia. El uso de segmentación UDP en RSVP-TE y de TCP en CR-LDP, acarrea diferencias en lo que se denomina estado del LSP. Además existen características de cada uno de los objetos en RSVP que agregan funcionalidades propias a cada protocolo. Los resultados presentan el retardo, el throughput y el jitter, para cada protocolo de señalización, haciendo uso de la herramienta de simulación GLASS.

\section{Evaluation of Signaling Protocols in Generalized Multiprotocol Label Switching (GMPLS) Capable of Switching Packets and Wavelength}

\begin{abstract}
In this paper, RSVP-TE and CR-LDP signaling protocols in the Generalized Multiprotocol Label Switching (GMPLS) are evaluated based on the design parameters of the physical layer, and packets and wavelength switching. In the simulations, of the signaling protocols the results do not present important discrepancies. The use of the UDP segmentation in RSVP-TE and of TCP in CR-LDP, present differences in the so-called LSP state. Additionally, some characteristics of each of the objects in RSVP that add other functionalities to each protocol. The results show the delay, throughput and jitter, for each signaling protocol, using the GLASS simulation tool.
\end{abstract}




\section{INTRODUCCIÓN}

Inicialmente, cuando se desarrolló la tecnología Multiprotocol Label Switching (MPLS), el objetivo era que la tecnología permitiera la conmutación de paquetes de una forma más rápida y eficiente que las tecnologías existentes para la época, como lo eran el Modo de Transferencia Asíncrono (ATM) y Frame Relay, pero sin dejarlas de lado (Piedrahita, 2012; Davie y Farrel, 2008; Ghein 2007; Guevara, 2011; López, et al., 2012). La solución para esto fue realizar una conmutación de etiquetas que tuviera todas las ventajas a nivel de enrutamiento IP y fuera independiente del protocolo utilizado a nivel del enlace de datos (Capa 2). Esta solución fue exitosa y la mayoría de proveedores de servicios a nivel mundial la implementaron (Rosen, et al., 2001). Adicionalmente, MPLS trajo consigo grandes beneficios a nivel de servicios como lo son las Redes Privadas Virtuales (VPNs), los túneles, entre otros (Knight y Lewis, 2004; Salcedo, 2012).

Desde hace aproximadamente una década la Internet Engineering Task Force (IETF), y diferentes fabricantes han trabajado en la arquitectura de GMPLS, que permite un control más general del nivel físico (Capa 1) con las redes MPLS (Yamanaka, et al., 2005; Farrel, 2006; Ming, 2008). GMPLS gestiona múltiples tecnologías de conmutación: conmutación de paquetes, conmutación de capa 2, conmutación de TDM (Multiplexación por división de tiempo), conmutación de longitud de onda y conmutación de fibra; mientras que MPLS maneja la conmutación de etiquetas. Con Generalized Multiprotocol Label Switching (GMPLS), se han hecho una serie de extensiones a los protocolos ya desarrollados en MPLS, junto con nuevos protocolos, que permiten al plano de control, datos y gestión, administrar y ofrecer nuevos servicios (Mannie, 2004). GMPLS permite a los operadores diseñar sus redes de acuerdo a los servicios necesitados; ya que a diferencia de MPLS soporta diferentes tipos de conmutación a velocidades ópticas, lo que permite incluir tecnologías como SDH/SONET y WDM (Multiplexación por división de longitud de onda), con un control más generalizado desde la capa 1 hasta la capa 3.El enfoque de este trabajo está limitado al plano de control, más específicamente a las tareas de señalización.

La señalización en GMPLS, se define como la tarea de establecer una ruta llamada Label Switched Path (LSP), entre dos nodos de la red, que permiten realizar el intercambio de etiquetas (Farrel, 2006). Esta señalización se puede realizar de dos maneras estática o dinámica, donde la señalización estática requiere la configuración manual del LSP en cada nodo, y la señalización dinámica requiere un protocolo de señalización (Berger, 2003). Los protocolos de señalización han sido objeto de análisis en las redes MPLS y GMPLS (Berger, 2003; lqbal, et al., 2005), debido a que han sufrido constantes evoluciones, entre estos se encuentran el Constraint-Routed Label Distribution Protocol (CR-LDP) (Ashwood y Berger, 2003), Resource Reservation Protocol Traffic Engineering (RSVP-TE) (Berger, 2003) y Border Gateway Protocol (BGP), cada uno con su propias características que afectan o benefician las diferentes variables en una red.

Entre los trabajos desarrollados en el área se destacan: (Molnar y Vlcek, 2010), en el que se presenta resultados sobre la eficiencia de los mecanismos de soporte de QoS disponibles en MPLS, para diferentes tipos de tráfico. En (Zheng y Mouftah, 2003), se propone una estructura para el soporte de servicios de reserva anticipada en GMPLS, aquí se incluye un modelo de reserva de longitudes de onda y un modelo de control de señalización. En (Sambo, et al., 2009), se exponen dos enfoques basados en el protocolo de enrutamiento y el protocolo de señalización, para diseminar la información del regenerador en el plano de control GMPLS y en (Casellas, et al., 2013), se diseña y valida un plano de control para GMPLS. En este documento se realiza una evaluación de protocolos de señalización de GMPLS, con el fin de analizar las posibles diferencias a nivel de parámetros de QoS.

\section{PROTOCOLOS DE SEÑALIZACIÓN}

La arquitectura MPLS define los protocolos de distribución de etiquetas como un conjunto de procedimientos por los cuales un Label Switching Router (LSR) informa a otro el significado de las etiquetas usadas para enviar tráfico entre y a través de ellos. Existen varios tipos de protocolos para hacer esta función como: Tag Distribution Protocol (TDP) (Davie y Farrel, 2008), Label Distribution Protocol (LDP), Resource Reservation Protocol (RSVP) y BGP (Ghein 2007).

En GMPLS, el diseño original incluye características de ingeniería de tráfico y enrutamiento restringido desde su origen (Mannie, 2004; Osborne y Simha, 2002). Para las labores de señalización se propuso dos protocolos, como son RSVP-TE y CR-LDP. A partir del año 2003, debido a una decisión impulsada por los fabricantes de equipos, la IETF decidió dar mayor enfoque al protocolo RSVP-TE para la señalización de GMPLS: tal como se refleja en estudios referentes a los LSPs, tráfico Inter-Dominio, entre otros (Farrel, et al., 2008; Ayyangar, et al., 2009); sin embargo CR-LDP se ha mantenido debido a que en MPLS el protocolo LDP se continua usando (Andersson, et al., 2007). 


\section{Constraint-Routed Label Distribution Protocol en GMPLS}

El enrutamiento explícito en LDP, se realiza cuando el Label Edge Router (LER) de origen dispone de los nodos por los cuales se construye el LSP a través de los mensajes Label Request que indican cuales nodos forman la trayectoria del LSP desde el LER de entrada hasta el de salida. El funcionamiento de CR-LDP es bastante similar a un enrutamiento explicito salto a salto, pero se agregan ciertas particularidades, que son nombradas a continuación:

El mensaje Label Request, especifica de forma explícita que rutas van a ser utilizadas. Las rutas explícitas reservan recursos utilizando características del enlace como son: Tasa de Datos Pico (PDR), Tasa de Datos Comprometida (CDR), Tamaño de Ráfaga Pico (PBS), Tamaño de Ráfaga Comprometida (CBS), frecuencia y peso. PDR y CDR describen las restricciones de velocidad de la ruta. PBS y CBS determinan los tamaños máximos de las trazas. La frecuencia específica que garantiza el CDR y el peso, establecen prioridades relativas en cada LSR para múltiples LSPs cuando hay congestión o poca capacidad disponible. Existe también la opción de negociar los recursos requeridos. Cuando el LSR no cuenta con los recursos existentes, reserva los recursos disponibles y los propaga por los Label Request.

El enrutamiento basado en restricciones ó Ingeniería de Tráfico (TE) en CR-LDP o RSVP-TE, es un mecanismo que busca sacar el mayor provecho de los recursos de la red, y para ser soportado se extendieron los protocolos de enrutamiento, tal como Open Shortest Path First-Traffic Engineering (OSPFTE), que es un protocolo de control para la diseminación de la topología y los atributos TE de los enlaces y nodos de la red de transporte. Usando dicha información cada instancia del plano de control es capaz de construir la ruta detallada de la red, conociendo atributos que permiten realizar cálculo de rutas que posteriormente se establecerán vía el protocolo de señalización. EI OSPF convencional solo considera para su métrica el costo de cada enlace para calcular la ruta más corta, usualmente también se consideran la capacidad instalada y/o la distancia física de cada enlace, sin tener en cuenta la utilización del enlace o algún tipo de retroalimentación del estado de la red. Así, OSPF debe agregar nuevos LSA (Link State Advertisements) para definir la ruta más corta con enrutamiento basado en restricciones (CSPF) (Kompella y Yeung, 2003). CR-LDP en GMPLS somete al LSP a ciertas modificaciones, entre las más importantes se encuentran (Ashwood y Berger, 2003): i) LSPs bidireccionales; ii) Procedimiento para la modificación de recursos; iii) Re-enrutamiento de los LSPs; iv) Manejo de prioridades; y v) Modificaciones en caso de falla.

\section{Resource Reservation Protocol Traffic Engineering en GMPLS}

RSVP surge a partir del modelo de servicios integrados (IntServ), que GMPLS está diseñado para instalar y mantener la información de estado de reserva en cada router de un LSP, esto con el fin de tener unas cualidades específicas del servicio de red para aplicaciones particulares. El protocolo RSVP basa su funcionamiento en los protocolos de enrutamiento IP, los cuales deciden que ruta deberá tomar el LSP (Berger, 2003).

Acerca del funcionamiento de RSVP, sus principales mensajes son PATH y RESV, el proceso da inicio en el momento que el nodo LER origen envía el mensaje PATH al nodo LER destino, especificando los requerimientos de reserva del flujo de tráfico. Luego, el LER destino envía un RESV al LER origen confirmando la reserva de los recursos. Cada uno de los mensajes en RSVP-TE contiene objetos que caracterizan la solicitud del mensaje, entre los más importantes están: Session, el cual identifica la dirección del flujo del tráfico mencionando el nodo fuente y destino; Sender_Template y Sender_Tspec, que es usado por los nodos intermedios para calificar el nodo origen y especificar el tráfico dentro del mensaje PATH; Filter-spec, FlowSpec y style, son utilizados por el mensaje RESV para definir la reserva del tráfico, especificar los requerimientos de recursos y diseñar el estilo de reserva.

\section{Diferencias entre los protocolos de señalización en GMPLS}

Las principales diferencias entre los protocolos de señalización CR-LDP y RSVP-TE son con respecto a su funcionamiento, estructura de mensajes, reacción al momento de fallas, manejo de tráfico, entre otras (Iqbal, et al., 2005; Brittain y Farrel, 2000). El protocolo de distribución de etiquetas, RSVP no está orientado a la conexión ya que usa como base para su funcionamiento el protocolo IP y UDP para el intercambio de mensajes; a diferencia del protocolo CR-LDP que usa UDP para descubrir a sus pares y TCP para distribuir las etiquetas (Farrel, 2006).

RSVP-TE es identificado como un protocolo de estado suave, ya que después de iniciar el proceso de LSP, los mensajes de actualización deben ser periódicamente intercambiados para notificar a los demás LSR que la conexión todavía es requerida. Esto permite a RSVP-TE actualizar de una manera rápida los cambios hechos para el enrutamiento, lo cual puede significar una ventaja al momento de una falla (Iqbal, et al., 
2005). Los mensajes de actualización de estado ayudan a mantener la sincronización entre los nodos adyacentes; sin embargo, estas actualizaciones periódicas implican una sobrecarga de procesamiento, lo que representa una de las debilidades en el protocolo y podría presentar problemas de escalabilidad. Una solución para reducir los mensajes de actualización es agrupar varios mensajes en uno solo, y de esta forma reducir la sobrecarga.

CR-LDP es referido como un protocolo de estado fuerte, lo que significa que toda la información es intercambiada en un tiempo inicial de configuración y ninguna información adicional es necesaria entre los nodos hasta que el LSP se encuentre sin conexión. Además, no se requiere que los LSRs actualicen el estado de su LSP después de configurado, esto con base en el uso que CR-LDP le da al protocolo TCP para el transporte de los mensajes de control. Cuando el sistema de gestión de red u otras entidades determinan que el LSP ya no es requerido, se intercambian algunos mensajes notificando a todos los nodos que los recursos deben ser liberados. Por tanto, CR-LDP presenta una menor carga en la señalización que RSVP-TE, debido a que no requiere mensajes de actualización de estado; sin embargo, una vez la actualización de mensajes es implementada en RSVP-TE, la carga en el protocolo se disminuye (Brittain y Farrel, 2000).

\section{METODOLOGÍA}

Para la evaluación de los protocolos de señalización en redes GMPLS, es necesario tener en cuenta parámetros de diseño, tales como selección de la fibra óptica, atenuación, refracción del material y cálculo del retardo

\section{Selección de la fibra óptica}

Una red GMPLS puede cubrir un área geográfica extensa, por tanto, se decidió utilizar una fibra óptica monomodo. Además, se debe definir una longitud de onda para su operación junto con un núcleo y revestimiento.

\section{Atenuación}

Las pérdidas sobre la fibra típicamente dependen de factores como la atenuación, que a su vez están directamente relacionados con la longitud de onda que se usa para transmitir. A medida que la longitud de onda aumenta, la atenuación por kilómetro $(\mathrm{dB} / \mathrm{km})$ disminuye. Para este caso se decide utilizar una longitud de onda de $1550 \mathrm{~nm}$ con pérdidas de $0,19 \mathrm{~dB} / \mathrm{km}$. Adicionalmente, esta longitud de onda requiere el uso de amplificadores de fibra de erbio dopado, los cuales ofrecen un alto desempeño (Cisco, 2008).

\section{Refracción del material}

La dispersión en las fibras ópticas es el fenómeno que describe el proceso por el cual la señal electromagnética propagada en un medio físico es degradada debido a las diferentes características de la señal de onda, generando diferentes velocidades de propagación dentro del medio físico. El índice de refracción varía con la longitud de onda (Russo, et al., 2011), esta variación es referida como dispersión (Borella, et al., 1997).

\section{Cálculo del retardo}

Para determinar el retardo de grupo $T g$, el cual es el tiempo de tránsito requerido por la potencia óptica para recorrer una distancia $L$, se usa la ecuación 1:

$T_{g}=\frac{L}{V_{g}}$

En donde $V g$ es la velocidad de grupo, que se puede hallar a partir de la ecuación 2:

$V_{g}=\frac{C}{n}$

Aquí C es la velocidad de la luz y $n$ el índice de refracción del material. 
Para tener un estimativo del retardo que generan los materiales sobre la fibra óptica, se tomó su índice de refracción a una longitud de onda de 1550nm y a diferentes distancias. En la Tabla 1 se puede observar los diferentes retardos hallados con las ecuaciones (1) y (2).

Tabla 1: Retardos de materiales de fibra óptica.

\begin{tabular}{|c|c|c|c|c|}
\hline Material & Distancia $(\mathrm{km})$ & $\begin{array}{l}\text { Índice de } \\
\text { Refracción }\end{array}$ & $\begin{array}{l}\text { Longitud de } \\
\text { Onda (nm) }\end{array}$ & $\begin{array}{c}\text { Retardo } \\
(\mu s)\end{array}$ \\
\hline \multirow{3}{*}{ Polimetilmetacrilato } & 1 & \multirow{3}{*}{1.44} & \multirow{3}{*}{1550} & 4.8 \\
\hline & 50 & & & 240 \\
\hline & 100 & & & 480 \\
\hline \multirow{3}{*}{ Dióxido de silicio } & 1 & \multirow{3}{*}{1.45} & \multirow{3}{*}{1550} & 4.835 \\
\hline & 50 & & & 241.75 \\
\hline & 100 & & & 483.5 \\
\hline \multirow{3}{*}{$\begin{array}{l}\text { Silicio dopado con } \\
\text { Germanio }\end{array}$} & 1 & \multirow{3}{*}{1.48} & \multirow{3}{*}{1550} & 4.933 \\
\hline & 50 & & & 246.65 \\
\hline & 100 & & & 493.3 \\
\hline
\end{tabular}

\section{RESULTADOS}

Para la evaluación de los protocolos de señalización en el diseño se utiliza la topología de red de la Fig. 1, que fue implementada mediante el simulador GLASS, que cuenta con un diseño modular de varios componentes: una capa física y lógica, protocolos de enrutamiento, restauración a fallas y algoritmos de asignación de longitudes (Muthuraj y Sreenath, 2013). En la simulación se tuvieron en cuenta características propias de una red convencional, como lo son, las posibles fallas en los enlaces, los parámetros físicos que pueden afectar el enlace óptico y un tráfico aleatorio. A nivel protocolar, se definió un algoritmo de enrutamiento, las características del tráfico que se genera entre el cliente y el servidor, y la implementación del protocolo RSVP-TE y CR-LDP (Piedrahita, 2012).

Durante la simulación se emplea una frecuencia de muestreo de $20 \mathrm{MHz}$, el tiempo total de la simulación es de 400s y la red tiene un tiempo de convergencia de 40s, tiempo empleado por OSPF. Los LSRs hacen uso de la conmutación de paquetes y la conmutación de longitud de onda, los 4 LSPs se definen con un enrutamiento explicito como el mostrado en la Fig. 1.

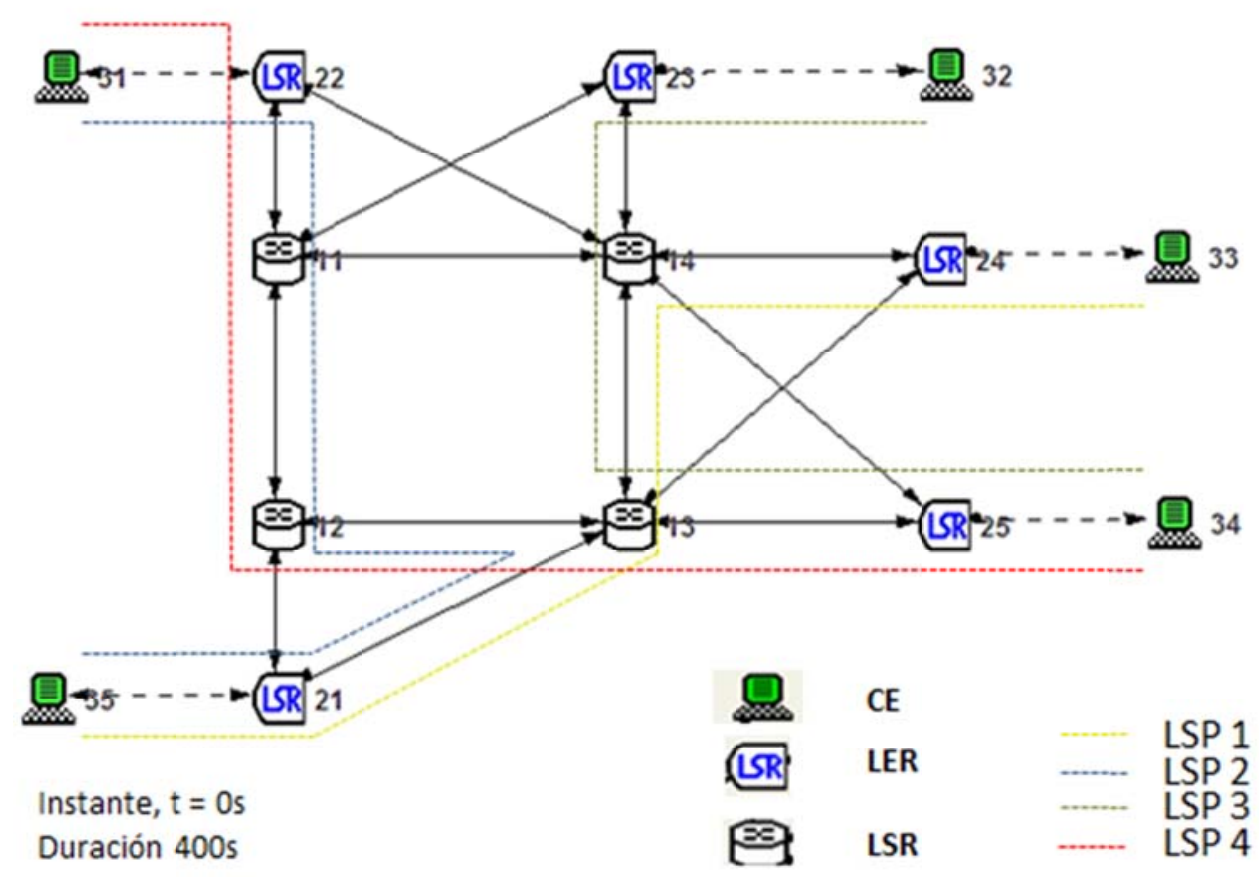

Fig. 1: Topología de red. 
En los esquemas de protección de los LSPs se configuran dos fallas en la red las cuales se ejecutaron en el tiempo de simulación $t=150$ s donde se afecta el enlace entre los nodos 11 y 12 y en el instante $t=300$ s se afecta el enlace entre los nodos 13 y 14, como se observa en la Fig. 2. El mecanismo de protección implementado en la simulación fue de tipo 1:1(que usa dos enlaces, dos nodos y recursos disyuntos, aunque el envío de la información solo se hace a través de un solo enlace; ya que el canal de respaldo es utilizado únicamente en el momento que una falla ocurra), con enlaces compartidos, lo que indica que solo se envía tráfico por un solo enlace bien sea el principal o el secundario, y los enlaces son compartidos con tráfico agregado por los demás servicios.

Falla 1

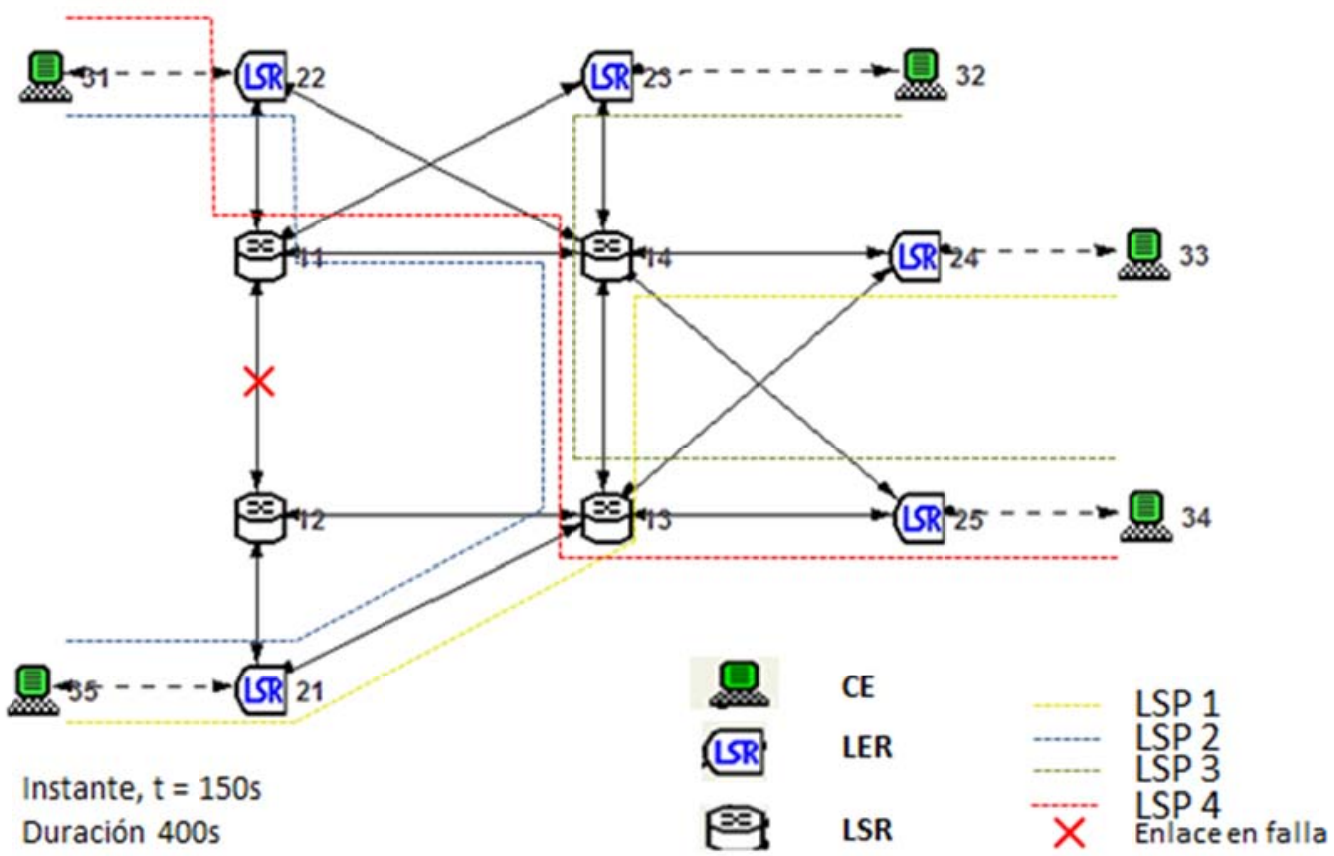

Falla 2

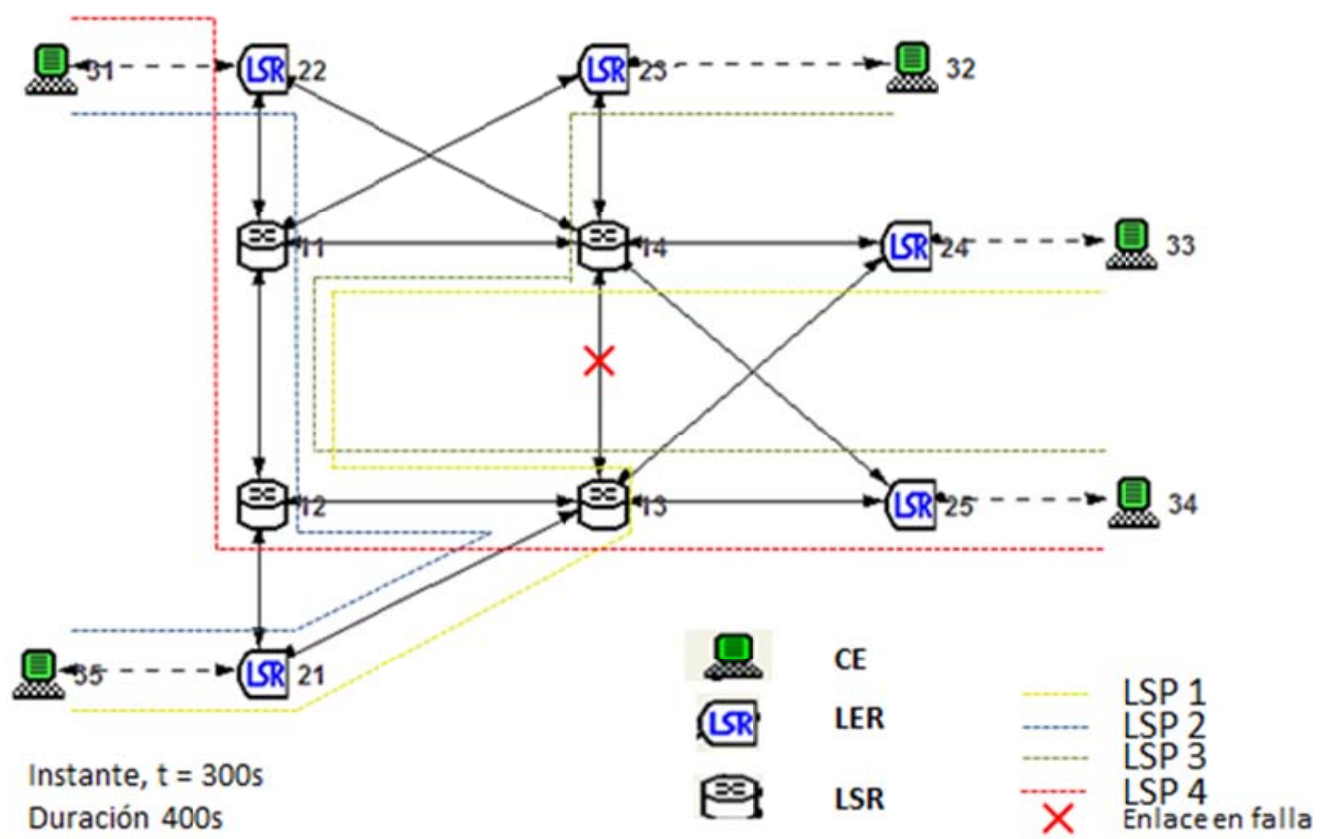

Fig. 2: Esquema de fallas. 


\section{Resultados del retardo}

Los resultados gráficos obtenidos por el simulador indican que las diferencias que puede generar el uso arbitrario de un protocolo de señalización no inciden de forma considerable en el retardo que pueda tener el envío o recepción de la información. Así mismo, para las fallas que se presentan en los instantes de tiempo t $=150 \mathrm{~s}$ y $\mathrm{t}=300 \mathrm{~s}$ existe un incremento en el retardo de los LSPs, por tanto el retardo en los LSPs primarios es menor que el de sus respaldos, debido a que se determina una nueva ruta para los LSPs, diferente a la óptima establecida inicialmente, lo cual conlleva a que existan enlaces con retrasos mayores.

En la Fig. 3 se observa el retardo generado por los cuatro LSPs al utilizar los dos protocolos de señalización. En la Fig. 1 a los 300s, resultado de la medición del retardo, se presenta un incremento en el retardo, en los LSP1 y LSP3, de aproximadamente el doble del tiempo; sin embargo en los LSP2 y LSP4 el aumento del retardo es tan solo de $50 \mu \mathrm{s}$, como consecuencia de los diferentes retardos que presentan los enlaces.

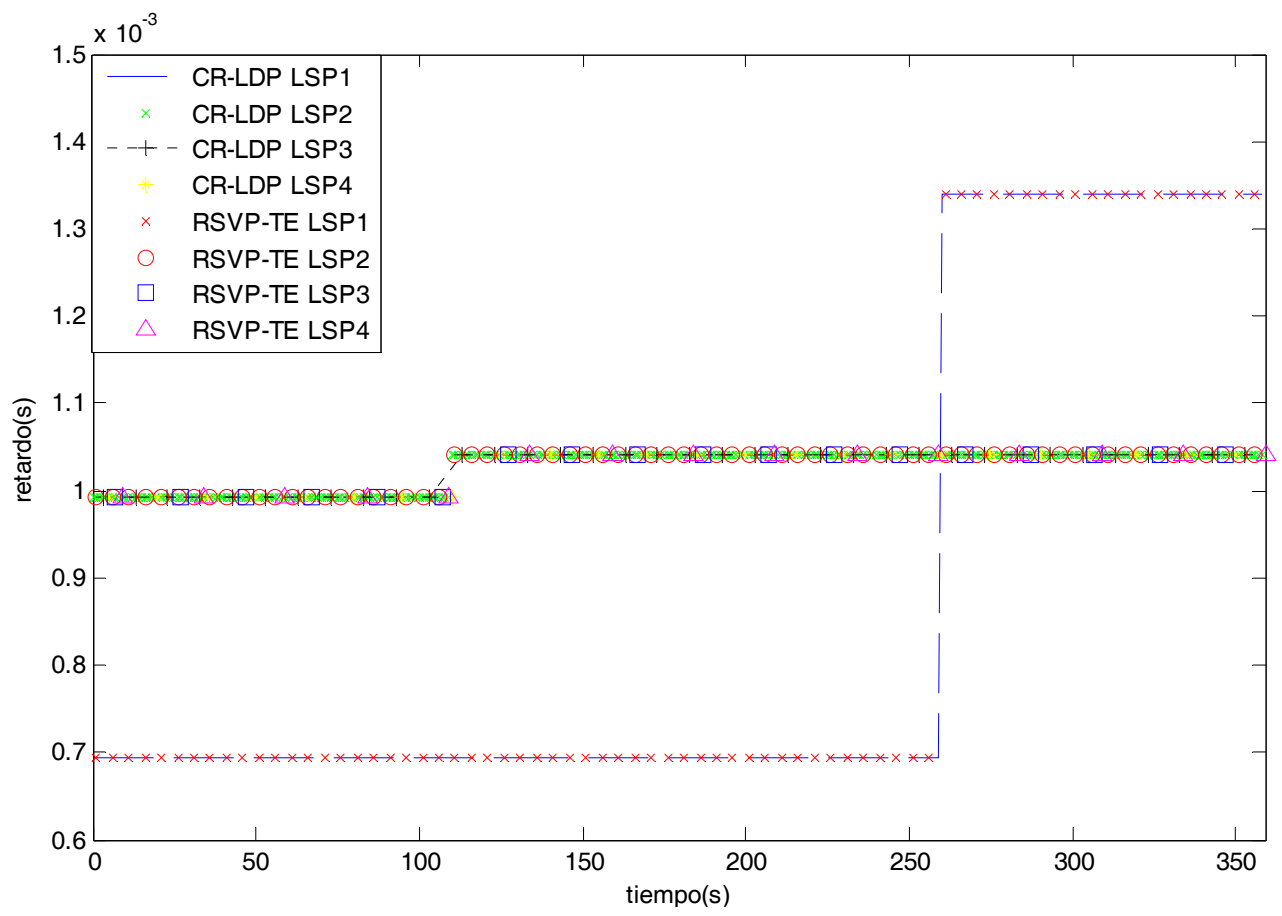

Fig. 3: Resultados del retardo.

\section{Resultados del Throughput}

El protocolo RSVP-TE alcanza picos de transferencia más altos que el protocolo CR-LDP para los diferentes LSPs, incluyendo la carga que se intercambia con los mensajes de control, tal como se exhibe en la Fig. 4. Los parámetros configurados dentro de la simulación para los LSPs son: 1) PDR (Peak Data Rate): El valor que definido es 625000 Bps que corresponde a la máxima capacidad que puede tener la interfaz de los CE (Equipos de Usuario); 2) PBS (Peak Burst Size): 10000 Bytes; 3) CDR (Commited Data Rate): corresponde a la capacidad acordada o contratada para el LSP, en la simulación el valor definido es de 40000 Bps; 4) CBS (Commited Burst Size): 10000 Bytes; y 5) EBS (Excess Burst Size): 0 Bytes.

A pesar del gran número de picos que se observan en los LSPs con el uso de RSVP-TE, el tráfico promedio enviado por los dos protocolos de señalización es muy cercano entre sí. La mayor diferencia de tráfico se presenta en el LSP3 (Fig. 4c), en donde CR-LDP obtiene un valor promedio de 40,133 KB/s y RSVP-TE de $40,143 \mathrm{~KB} / \mathrm{s}$. En general, esta leve diferencia se debe a que CR-LDP usa el protocolo TCP y RSVP-TE emplea el protocolo UDP.

\section{Resultados de la variación del retardo (Jitter)}

El jitter o variación del retardo, permite identificar las diferencias entre el tiempo de envío y recepción durante toda la transmisión. En la Fig. 5, se presenta el jitter de cada uno de los LSPs. Al emplear el protocolo CR-LDP, el jitter se incrementa a medida que el tiempo de simulación aumenta; sin embargo los valores que toma esta métrica de QoS durante la simulación siguen siendo bajos ya que oscilan hasta un máximo de $15 \times 10^{-15} \mathrm{~s}$, que es una adecuada diferencia de tiempo para muchas aplicaciones. 


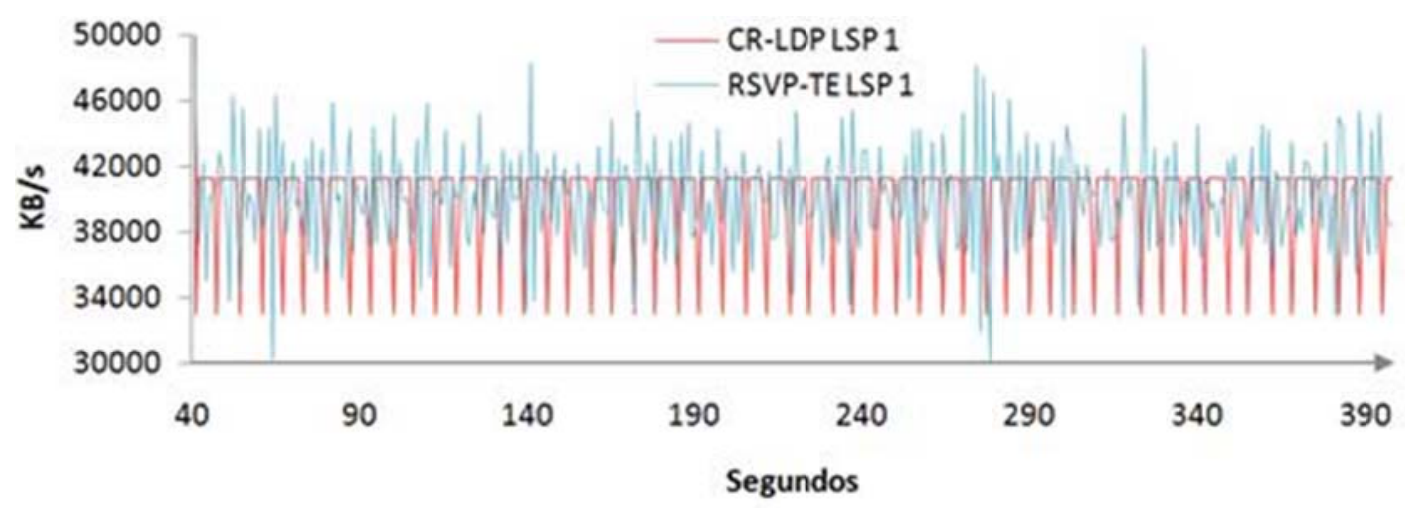

a)

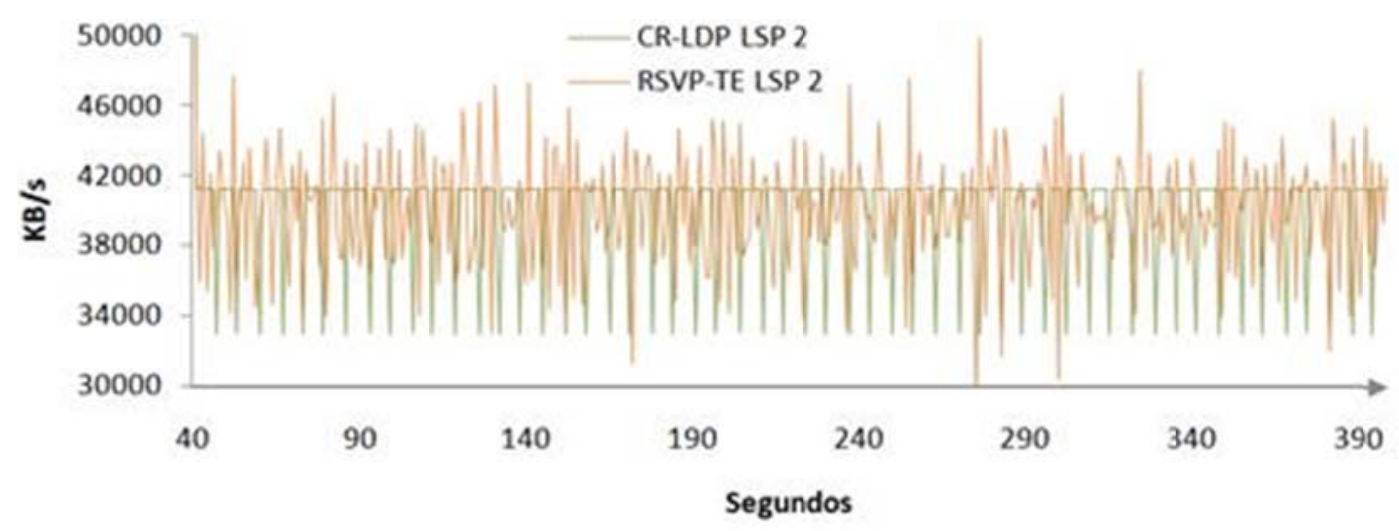

b)

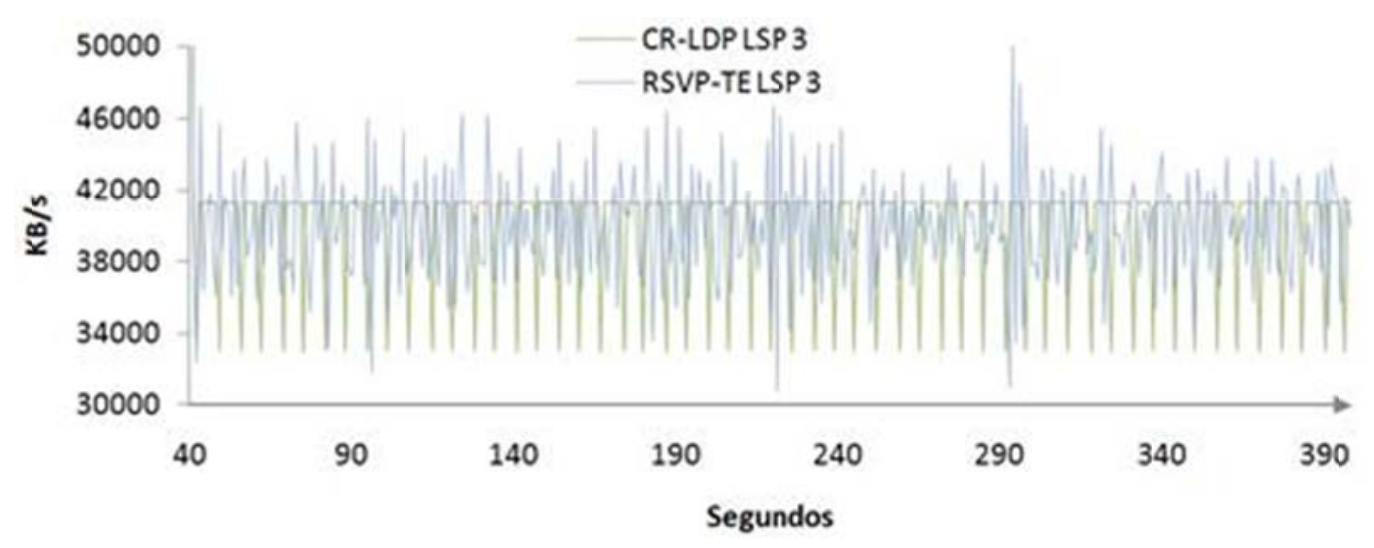

c)

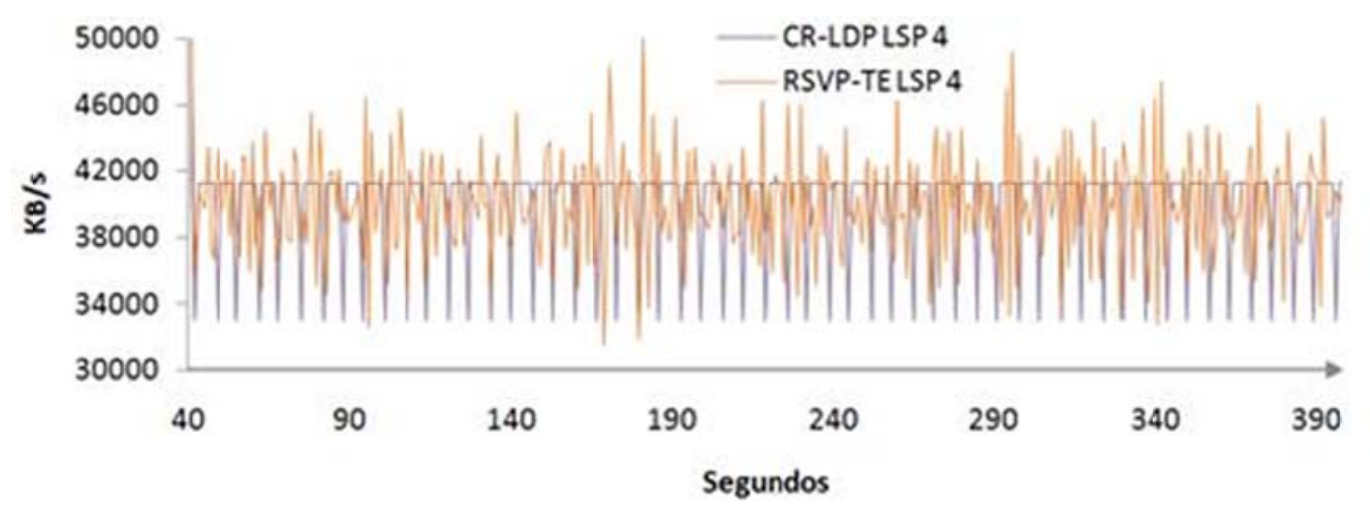

d)

Fig. 4: Resultados de throughput. 


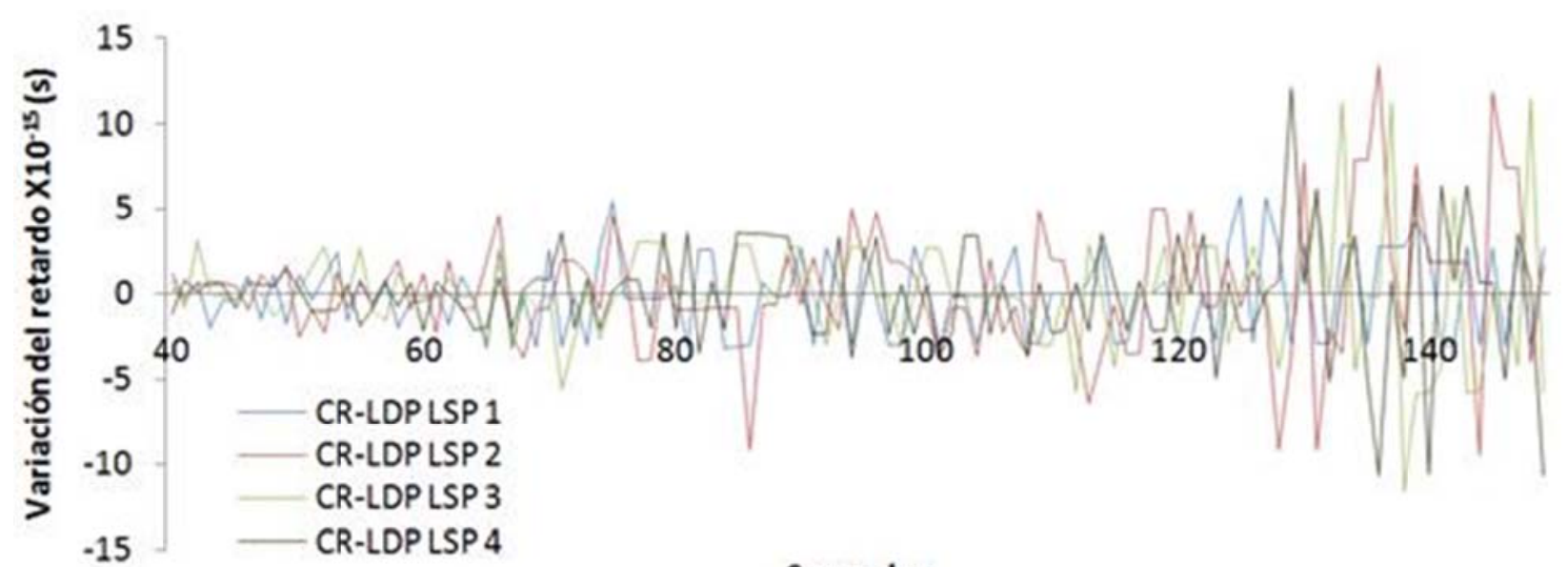

\section{Segundos}

Fig. 5: Resultados de Jitter para CR-LDP.

En la Fig. 6, se observa el jitter obtenido por los 4 LSPs con RSVP-TE, en este protocolo también se incrementa el jitter a medida que el tiempo transcurre. Adicionalmente, las fluctuaciones de la variación del retardo llegaron hasta $8 \times 10^{-15} \mathrm{~s}$, lo cual representa una ventaja para RSVP-TE.

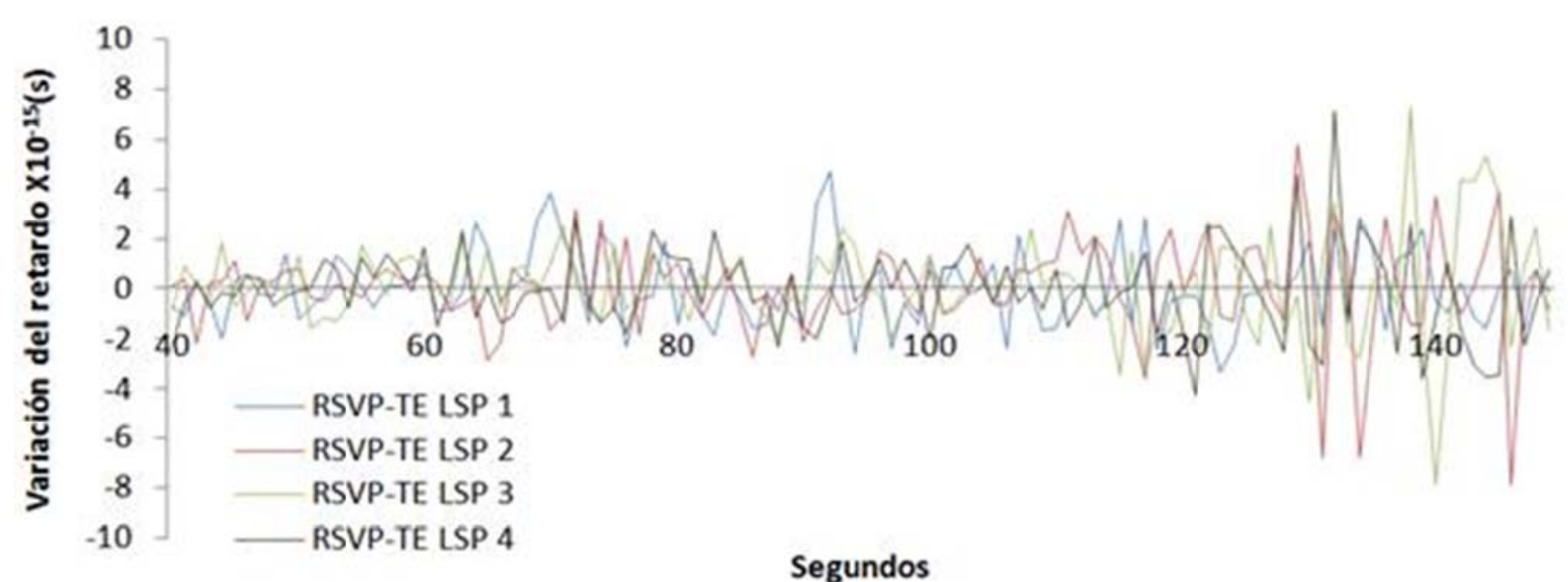

Fig. 6: Resultados de Jitter para RSVP-TE.

Los valores de jitter obtenidos en la simulación para los dos protocolos de señalización, son muy bajos y mucho menores a los valores que normalmente son acordados entre los usuarios y los proveedores de la red; aunque el valor aumenta a través del tiempo a causa de los retardos presentes en la red. En las Fig. 5 y 6 los resultados se presentan hasta el instante donde ocurre la primera falla, ya que posterior a esto, los valores de jitter aumentan de forma excesiva, lo cual no facilita el análisis.

\section{CONCLUSIONES}

Las métricas de QoS empleadas para la evaluación de los protocolos de señalización RSVP-TE y CR-LDP, permitieron identificar algunas diferencias entre ellos. Con respecto al jitter se evidenció que RSVP-TE tuvo un mejor desempeño con base en las variaciones que lograron un valor máximo alrededor de $\pm 10^{-16} \mathrm{~s}$; mientras que los valores obtenidos para CR-LDP presentaron un valor pico cercano a los $\pm 10^{-15} \mathrm{~s}$. Por otra parte, el throughput promedio que se registra en los LSPs posee unas diferencias no mayores a los 100Bps para cada prueba, lo que permite inferir que fue similar en los dos protocolos. En cuanto al retardo los resultados muestran diferencias del orden de $10^{-5} \mathrm{~s}$, que no evidencia cambios que afecten el comportamiento de los servicios. Por tanto, el uso de ambos protocolos de señalización (CR-LDP y RSVPTE) conlleva a un buen desempeño para los parámetros de QoS analizados. 
Al analizar las simulaciones de los protocolos de señalización los resultados no presentan mayor discrepancia; sin embargo en la estructura y funcionamiento de éstos no es notable esta similitud. Esencialmente, el uso de segmentación UDP en RSVP-TE y de TCP en CR-LDP, acarrea diferencias en lo que se denomina estado del LSP, además existen características de cada uno de los objetos en RSVP o en los TLVs (Type Length Value) de CR-LDP que agregan funcionalidades propias a cada protocolo.

\section{REFERENCIAS}

Andersson, L., I. Minei y B. Thomas, RFC 5036: LDP Specification, Technical Report (2007).

Ashwood, P. y L. Berger, RFC 3472: Generalized Multi-Protocol Label Switching (GMPLS) Signaling Constraint-based Routed Label Distribution Protocol (CR-LDP) Extensions, Technical Report (2003).

Ayyangar, A., D. Papadimitriou y A. Farrel, RFC 5420: Encoding of Attributes for MPLS LSP Establishment Using Resource Reservation Protocol Traffic Engineering (RSVP-TE), Technical Report (2009).

Berger, L., RFC 3471: Generalized Multi-Protocol Label Switching (GMPLS) Signaling Functional Description, Technical Report (2003).

Berger, L., RFC 3473: Generalized Multi-Protocol Label Switching (GMPLS) Signaling Resource Reservation Protocol-Traffic Engineering (RSVP-TE) Extensions, Technical Report (2003).

Borella, M., J. Jue, D. Banerjee, B. Ramamurthy y B. Mukherjee, Optical components for WDM lightwave networks, Proceedings of the IEEE: 85(8), 1274 -1307 (1997).

Brittain P. y A. Farrel, MPLS Traffic Engineering - A Choice Of Signaling Protocols, http://networktechnologies.metaswitch.com/download/crldprsvp.pdf, England, UK (2000).

Casellas, R., R. Munoz, J.M. Fabrega, M.S. Moreolo, R. Martinez, L. Lei, T. Tsuritani e I. Morita, Design and Experimental Validation of a GMPLS/PCE Control Plane for Elastic CO-OFDM Optical Networks, IEEE Journal on Selected Areas in Communications, 1(31), 49-61, (2013).

Cisco, Fiber Types in Gigabit Optical Communications, Technical Report (2008).

Davie, B. y A. Farrel, MPLS: Next Steps, 1ª edición. Elsevier, USA (2008).

Farrel, A. e I. Bryskin, GMPLS: Architecture and Applications, $1^{\text {a }}$ edición. Morgan Kaufmann, San Francisco, USA (2006).

Farrel, A., A. Ayyangar y JP. Vasseur, RFC 5151: Inter-Domain MPLS and GMPLS Traffic Engineering -Resource Reservation Protocol-Traffic Engineering (RSVP-TE) Extensions, Technical Report (2008).

GCO, Tutorial de Comunicaciones Ópticas, http://nemesis.tel.uva.es/images/tCO/index.htm, Valladolid, España (2004) .

Ghein, L., MPLS Fundamentals, 1ª edición. Cisco Press, Indianapolis, USA (2007).

Guevara, A., Nivel de desempeño en redes IPV4 con respecto a redes IPV6 con MPLS y RSVP, Tecnura: 15 (28), 123-133, (2011).

Iqbal, A., W. Mahmood, E. Ahmed y K. Samad, Evaluation of distributed control signaling protocols in GMPLS, Fourth International Conference on the Optical Internet, Chongqing, Chinese, May (2005).

Knight, P. y C. Lewis, Layer 2 and 3 Virtual Private Networks: Taxonomy, Technology and Standardization Efforts, Communications Magazine IEEE: 42(6), 124 - 131 (2004).

Kompella, K., D. Katz y D. Yeung, RFC 3630: Traffic Engineering (TE) Extensions to OSPF Version 2, Technical Report (2003).

López, D., C. Hernández y O. Salcedo, Redes Multidifusión utilizando Conmutación de Etiquetas Multiprotocolo y Estándares de Señalización, Información tecnológica, 23(6), 43-50, (2012). 
Mannie, E., RFC 3945: Generalized Multi-Protocol Label Switching (GMPLS) Architecture, Technical Report (2004).

Ming, H. Development trends of GMPLS control plane, Huawei: (42), 48-50, 2008.

Molnar, K. y M. Vlcek, Evaluation of Quality-of-Service Support in MultiProtocol Label Switching, Fifth International Conference on Systems and Networks Communications, Nice, France, 22-27 August (2010).

Mukherjee, B. Optical WDM Networks, $1^{\text {a }}$ edición, Springer, New York, USA (2006).

Muthuraj, K. y N.Sreenath, Optical internet: a comparative assessment on TCP over OBS networks simulation tools, International Journal of Advanced Computer Technology: 2(1), 1-9 (2013).

Osborne, E. y A. Simha, Traffic Engineering with MPLS, $1^{\text {a }}$ edición. Cisco Press, Indianapolis, USA (2002).

Piedrahita, E., Evaluación de los Protocolos de Señalización en el Plano de Control en Redes GMPLS, Tesis de Maestría, Universidad Distrital Francisco José de Caldas, Facultad de Ingeniería, Bogotá, Colombia, (2012).

Rosen, E., A. Viswanathan y R. Callon, RFC 3031: Multiprotocol Label Switching Architecture, Technical Report (2001).

Russo, N., S. Noriega y R. Duchowicz, Implementación de Sistema Óptico para Grabado de Redes de Bragg en Fibra Óptica, Información tecnológica, 22(2), 121-130, (2011).

Salcedo, O., L. F. Pedraza y M. Espinosa, Evaluación de redes MPLS/VPN/BGP con rutas reflejadas, Tecnura: 16 (32), 107-116, (2012).

Sambo, N., N. Andriolli, A. Giorgetti, L. Valcarenghi, I. Cerutti, P. Castoldi y F. Cugini, GMPLS-controlled dynamic translucent optical networks, Network IEEE , 3(23), 34-40, (2009).

Yamanaka, N., K. Shiomoto y E. Oki, GMPLS Technologies: Broadband Backbone Networks and Systems, $1^{\text {a }}$ edición. CRC Press, Boca Raton, USA (2005).

Zheng, J. y H.T. Mouftah, A framework for supporting advance reservation service in GMPLS-based WDM networks, IEEE Pacific Rim Conference on Communications, Computers and signal Processing, Victoria, Canada, 28-30 August (2003). 
